\title{
EVALUATION OF CIMMYT WHEAT LINES UNDER EGYPTIAN FIELD CONDITIONS TO IDENTIFY NEW SOURCES OF RESISTANCE TO LEAF RUST
}

\author{
aWalid M. El-Orabey*, bHosam M. Awad, cSabry I. Shahin, dYasser A. El-Gohary \\ a Plant Pathology Research Institute, ARC, P.O. Box: 12619, Giza, Egypt. \\ b Agricultural Botany Department, Faculty of Agriculture, Menoufia University, Egypt. \\ c Sustainable Development of Environment and its Projects Dept., Environmental Studies and Research Institute, \\ University of Sadat City, Sadat City, Egypt. \\ d Wheat Research Department, Field Crops Research Institute, ARC, Giza, Egypt.
}

\section{A R T I C LE I N F O}

\section{Article history}

Received: February 16, 2020

Revised: May 24, 2020

Accepted: August 05, 2020

\section{Keywords}

Wheat

Leaf rust

Adult plant resistance

Promising lines
A B S T R A C T

Leaf rust caused by Puccinia triticina Eriks. is a fungal disease of wheat (Triticum aestivum L.), which causes considerable yield loss. Host resistance is the most effective and economical method to minimize yield losses caused by leaf rust. The current research was planned to evaluate the response of 93 wheat genotypes lines selected from 716 wheat genotypes delivered to Egypt by International Maize and Wheat Improvement Center (CIMMYT). These genotypes were evaluated against leaf rust resistance under field conditions at two locations i.e. Behira governorate (Itay El-Baroud Agricultural Research Station) and Menoufia governorate during three successive growing seasons i.e. 2017/2018, 2018/2019 and 2019/2020. Results of the current study showed that 47 wheat genotypes were resistant and had the lowest values of final rust severity (FRS \%), average coefficient of infection (ACI) and area under disease progress curve (AUDPC). Also, these genotypes showed desirable/acceptable relative resistance index (RRI) at the two locations during the three growing seasons of the study. Therefore, we can select these genotypes as resistant lines in the breeding program for the resistance of leaf rust.

Corresponding Author: Walid M. El-Orabey

Email: walid_elorabey2014@hotmail.com

(C) The Author(s) 2020.

\section{INTRODUCTION}

Wheat leaf rust caused by Puccinia triticina Eriks. is the most common, widespread, and devastating disease in Egypt and worldwide. Leaf rust has the potential to cause losses up to $50 \%$ and because of its frequent and widespread occurrence, leaf rust probably results in greater total annual losses worldwide than stem and stripe rusts (Huerta-Espino et al., 2011). In Egypt, grain yield loss due to artificial leaf rust has reached $32 \%$ in the susceptible wheat cultivars that are cultivated under experimental field conditions favorable to disease incidence and development (Shahin and ElOrabey, 2016; El-Orabey et al., 2017).
The preferable and most economical method for controlling wheat rusts is the utilization of genetic resistance. It is the most effective, economically safe, and eco-friendly approach, as this method eliminates the need to use fungicides and reduces the cost of production. The need is to identify those cultivars with resistant sources to be suggested as the fittest for the cultivation in the diseased areas of the country keeping in view different ecological zones. The screening is considered as the best and the cheapest way to identify these cultivars of wheat which show resistance against leaf rust (Anwar et al., 2019).

To contain the disease outbreak, Egyptian wheat 
breeders have developed several rust-resistant varieties in collaboration with pathologists and utilized advanced breeding materials. Despite the development of rust-resistant cultivars, the emergence of newer types of virulent races had led to a breakdown of resistance. Hence, the current breeding strategy warrants pyramiding disease resistance genes for all the three rusts in commercially released high yielding varieties (Tyagi et al., 2014). At present, more than 80 different leaf rust resistance genes and QTL spread throughout the A, B, and D wheat genomes have been identified and cataloged (Sapkota et al., 2018). Resistance imparted by many of these genes has either broken down or been lost due to emerging newer races with higher virulence and poor management of germplasm. It is, therefore, desirable that germplasm exhibiting resistance through non-specific interaction is used in breeding programs rather than germplasm exhibiting only specific interaction (McIntosh et al., 1995). Hence, screening of large number of germplasm accession is essential to identify newer and diverse sources of resistance to new races/pathotypes of wheat rusts (Daetwyler et al., 2014).

Screening entire cultivated wheat collections from gene banks in hotspots to identify trait-specific germplasm assumes unprecedented significance in this context. Such screening may bring to light new genes and genetic combinations in adapted genetic backgrounds for use as a source of resistance in future breeding programs. Such trials have been conducted in the past, including global initiatives screening over 200,000 wheat lines for resistance to Ug99 in Kenya (Singh et al., 2002) and a national effort in the screening of wheat germplasm for stripe rust tolerance in Pakistan (Bux et al., 2012). Germplasm conserved in genebanks (including crop wild relatives) is always a potential source of resistance genes that can be utilized efficiently to incorporate multiple disease resistances into popular cultivars (Jin and Singh, 2006). The objective of the present study was to identify disease-resistant wheat germplasm to leaf rust based on screening at disease hotspots and evaluate resistant germplasm against different leaf rust races under artificial epiphytotic conditions.

\section{MATERIALS AND METHODS}

\section{Plant Materials}

A total of 716 wheat genotypes in four sets were provided to Egypt by International Maize and Wheat
Improvement Center (CIMMYT), Mexico, through the website (http://www.cimmyt.org/seed-request/\#wheat) including the wheat variety; Morocco (check for rust resistance) as a highly susceptible. The five sets of germplasm evaluated included (1) Elite Spring Wheat Yield Trial (ESWYT), (2) Stem Rust Resistance Screening Nursery (STEMRRSN), (3) International Spring Bread Wheat Screening Nursery (IBWSN), and (4) HighTemperature Wheat Yield Trial (HTWYT) consisting of $98,168,329$ and 121 entries, respectively. A total of 93 i.e. 26 genotypes from (ESWYT), 32 (STEMRRSN), 25 (IBWSN), and 10 (HTWYT) wheat germplasm were selected from 716 tested wheat genotypes which were selected according to their response for leaf rust resistance under field conditions. The pedigree of the tested genotypes is shown in Table (1).

\section{Field Testing}

The experiments of this study were carried out at two locations i.e. Behira governorate (Itay El-Baroud Agricultural Research Station) and Menoufia governorate (Shibin El-Kom) during 2017/2018, 2018/2019, and $2019 / 2020$ successive growing seasons. These experiments were conducted in randomized complete block design (RCBD) with three replicates. The tested wheat genotypes were sown in rows of $3 \mathrm{~m}$ long. The experiments were surrounded by a spreader area planted with a mixture of highly susceptible wheat genotypes to leaf rust. These genotypes were Triticum spelta sahariensis, Morocco, and Thatcher to spread rust inoculum. For field inoculation with leaf rust, the spreader plants were sprayed with a mist of water and dusted with a mixture of aggressive urediniospores of the prevalent and aggressive seven pathotypes i.e. TTTJT, PTTCT, PTTGS, PTTTT, TTTBT, TTTKT, and TTTTT (El-Orabey et al., 2018) mixed with a talcum powder at a ratio of 1: 20 (v/v) (spores : talcum powder).

Plants were dusted in the early evening (at sunset) before dew point formation on the leaves. The inoculation of all plants was carried out at the booting stage according to the method of Tervet and Cassell (1951). The urediniospores of leaf rust received from Wheat Research Diseases Department, Plant Pathology Research Institute, Agricultural Research Center, Egypt. To maintain crop stand/vigor normal agronomic practices including recommended fertilization dose and irrigation schedules were followed. 
Table 1: Pedigree of wheat genotypes used in this study.

\begin{tabular}{|c|c|c|c|}
\hline Line & Pedigree & Line & Pedigree \\
\hline 1 & ROLF07*2/3/PRINIA/PASTOR//HUITES & 48 & $\begin{array}{l}\text { 7846//2180/4/2*MILAN/KAUZ//PRINIA } \\
\text { /3/BAV92 }\end{array}$ \\
\hline 2 & $\begin{array}{l}\text { CNO79//PF70354/MUS/3/PASTOR/4/BAV92 } \\
\text { *2/5/FH6-1-7 }\end{array}$ & 49 & $\begin{array}{l}\text { BOW/VEE/5/ND/VG9144//KAL/BB/3/YACO/ } \\
\text { 4/CHIL/6/CASKOR/3/CROC_1/AE.SQUARROS } \\
\text { A (224)//OPATA/7/PASTOR// } \\
\text { MILAN/KAUZ/3/BAV92 }\end{array}$ \\
\hline 3 & KACHU \#1/KIRITATI//KACHU & 50 & $\begin{array}{l}\text { BOW/VEE/5/ND/VG9144//KAL/BB/3/YACO/ } \\
\text { 4/CHIL/6/CASKOR/3/CROC_1/AE.SQUARROSA } \\
\text { (224)//OPATA/7/PASTOR//MILAN/KAUZ/3/ } \\
\text { BAV92 }\end{array}$ \\
\hline 4 & $\begin{array}{l}\text { WBLL1*2/4/BABAX/LR42//BABAX/3/BABAX } \\
\text { /LR42//BABAX }\end{array}$ & 51 & $\begin{array}{l}\text { D67.2/PARANA 66.270//AE.SQUARROSA } \\
(320) / 3 / \text { CUNNINGHAM/4/VORB }\end{array}$ \\
\hline 5 & ATTILA*2/PBW65*2//MURGA & 52 & $\begin{array}{l}\text { D67.2/PARANA 66.270//AE.SQUARROSA } \\
(320) / 3 / \text { CUNNINGHAM/4/VORB }\end{array}$ \\
\hline 6 & $\begin{array}{l}\text { ROLF07*2/5/REH/HARE//2*BCN/3/CROC_1/ } \\
\text { AE.SQUARROSA (213)//PGO/4/HUITES }\end{array}$ & 53 & $\begin{array}{l}\text { H45/4/KRICHAUFF/FINSI/3/URES/PRL//BA } \\
\text { V92 }\end{array}$ \\
\hline 7 & ATTILA*2/PBW65*2//W485/HD29 & 54 & $\begin{array}{l}\text { EGA BONNIE } \\
\text { ROCK/4/MILAN/KAUZ//PRINIA/3/BAV92 }\end{array}$ \\
\hline 8 & $\begin{array}{l}\text { WBLL1*2/TUKURU//FN/2*PASTOR/3/FRET2 } \\
\text { /KIRITATI }\end{array}$ & 55 & $\begin{array}{l}\text { CNDO/R143//ENTE/MEXI_2/3/AEGILOPS } \\
\text { SQUARROSA } \\
\text { (TAUS)/4/WEAVER/5/2*JANZ/6/D67.2/ } \\
\text { PARANA 66.270//AE.SQUARROSA } \\
\text { (320)/3/CUNNINGHAM }\end{array}$ \\
\hline 9 & $\begin{array}{l}\text { NAC/TH.AC//3*PVN/3/MIRLO/BUC/4/2*PAS } \\
\text { TOR/5/KACHU/6/KACHU }\end{array}$ & 56 & $\begin{array}{l}\text { INQALAB } \\
\text { 91*2/KUKUNA/4/TC14/2*HTG//DUCULA/3/ } \\
\text { PRINIA }\end{array}$ \\
\hline 10 & $\begin{array}{l}\text { WAXWING/4/BL } \\
\text { 1496/MILAN/3/CROC_1/AE.SQUARROSA } \\
\text { (205)//KAUZ/5/FRNCLN }\end{array}$ & 57 & $\begin{array}{l}\text { KANZ/5/CNO79//PF70354/MUS/3/PASTOR/ } \\
\text { 4/BAV92/6/PRL/SARA//TSI/VEE\#5 }\end{array}$ \\
\hline 11 & $\begin{array}{l}\text { WBLL1*2/KURUKU/6/CNDO/R143//ENTE/M } \\
\text { EXI_2/3/AEGILOPS SQUARROSA } \\
\text { (TAUS)/4/WEAVER/5/2*JANZ/7/ } \\
\text { WBLL1*2/KURUKU }\end{array}$ & 58 & BABAX/KS93U76//BABAX/3/2*SOKOLL \\
\hline 12 & $\begin{array}{l}\text { UP2338*2/VIVITSI/3/FRET2/TUKURU//FRE } \\
\text { T2/4/MISR } 1\end{array}$ & 59 & ATTILA*2/PBW65*2//KACHU \\
\hline 13 & $\begin{array}{l}\text { TACUPETO } \\
\text { F2001*2/BRAMBLING//WBLL1*2/BRAMBLI } \\
\text { NG }\end{array}$ & 60 & ROLF07*2/3/PRINIA/PASTOR//HUITES \\
\hline 14 & $\begin{array}{l}\text { CNO79//PF70354/MUS/3/PASTOR/4/BAV92 } \\
* 2 / 5 / \text { FH6-1-7 }\end{array}$ & 61 & $\begin{array}{l}\text { CNO79//PF70354/MUS/3/PASTOR/4/BAV92 } \\
* 2 / 5 / \text { FH6-1-7 }\end{array}$ \\
\hline 15 & FRET2/TUKURU//FRET2/3/MUNAL \#1 & 62 & KACHU \#1/KIRITATI//KACHU \\
\hline 16 & FRET2/TUKURU//FRET2/3/MUNAL \#1 & 63 & PBW343*2/KUKUNA*2//FRTL/PIFED \\
\hline 17 & $\begin{array}{l}\text { GAN/AE.SQUARROSA } \\
(408) / / 2 * \text { OASIS/5*BORL95/3/ TACUPETO } \\
\text { F2001*2/BRAMBLING }\end{array}$ & 64 & $\begin{array}{l}\text { WBLL1*2/4/BABAX/LR42//BABAX/3/BABAX } \\
\text { /LR42//BABAX }\end{array}$ \\
\hline 18 & KIRITATI//ATTILA*2/PASTOR/3/AKURI & 65 & ATTILA*2/PBW65*2//MURGA \\
\hline 19 & KIRITATI//PRL/2*PASTOR/3/FRANCOLIN \#1 & 66 & $\begin{array}{l}\text { SUP152/4/BABAX/LR42//BABAX*2/3/KURU } \\
\text { KU }\end{array}$ \\
\hline 20 & BAJ \#1/3/KIRITATI//ATTILA*2/PASTOR & 67 & $\begin{array}{l}\text { QUAIU/5/FRET2*2/4/SNI/TRAP\#1/3/KAUZ* } \\
\text { 2/TRAP//KAUZ }\end{array}$ \\
\hline 21 & $\begin{array}{l}\text { WBLL1*2/BRAMBLING/3/KIRITATI//PBW65 } \\
\text { /2*SERI.1B }\end{array}$ & 68 & $\begin{array}{l}\text { TACUPETO } \\
\text { F2001*2/BRAMBLING//WBLL1*2/BRAMBLING }\end{array}$ \\
\hline 22 & WBLL1*2/KURUKU//SUP152 & 69 & $\begin{array}{l}\text { ROLF07*2/5/REH/HARE//2*BCN/3/CROC_1/ } \\
\text { AE.SQUARROSA (213)//PGO/4/HUITES }\end{array}$ \\
\hline 23 & $\begin{array}{l}\text { WBLL4/KUKUNA//WBLL1/3/WBLL1*2/BRA } \\
\text { MBLING }\end{array}$ & 70 & $\begin{array}{l}\text { NAC/TH.AC//3*PVN/3/MIRLO/BUC/4/2*PAS } \\
\text { TOR/5/KACHU/6/KACHU }\end{array}$ \\
\hline
\end{tabular}


Table 1: Cont...

\begin{tabular}{|c|c|c|c|}
\hline 24 & $\begin{array}{l}\text { FRET2*2/BRAMBLING/3/FRET2/WBLL1//TA } \\
\text { CUPETO F2001/4/WBLL1*2/BRAMBLING }\end{array}$ & 71 & $\begin{array}{l}\text { WAXWING/4/BL 1496/MILAN/3/CROC_1/AE. } \\
\text { SQUARROSA (205)//KAUZ/5/FRNCLN }\end{array}$ \\
\hline 25 & $\begin{array}{l}\text { WHEAR*2/3/FRET2/WBLL1//TACUPET0 } \\
\text { F2001 }\end{array}$ & 72 & TACUPETO F2001/BRAMBLING//KACHU \\
\hline 26 & $\begin{array}{l}\text { ALTAR 84/AE.SQUARROSA } \\
\text { (221)//3*BORL95/3/URES/JUN//KAUZ/4/WBL } \\
\text { L1/5/KACHU/6/KIRITATI//PBW65/2*SERI.1B }\end{array}$ & 73 & $\begin{array}{l}\text { SITE/MO//PASTOR/3/TILHI/4/WAXWING/KI } \\
\text { RITATI }\end{array}$ \\
\hline 27 & FRANCOLIN \#1*2/MUU & 74 & $\begin{array}{l}\text { ALTAR 84/AE.SQUARROSA } \\
\text { (221)//3*BORL95/3/ } \\
\text { URES/JUN//KAUZ/4/WBLL1/5/REH/HARE// } \\
\text { 2*BCN/3/CROC_1/AE.SQUARROSA } \\
(213) / / \text { PGO/4/HUITES }\end{array}$ \\
\hline 28 & FRANCOLIN \#1*2/KINGBIRD \#1 & 75 & ROLF07*2/3/PRINIA/PASTOR//HUITES \\
\hline 29 & $\begin{array}{l}\text { SERI.1B*2/3/KAUZ*2/BOW//KAUZ*2/4/KIN } \\
\text { GBIRD \#1 }\end{array}$ & 76 & $\begin{array}{l}\text { ROLF07*2/4/CROC_1/AE.SQUARROSA } \\
(205) / / \text { BORL95/3/2*MILAN }\end{array}$ \\
\hline 30 & HUIRIVIS \#1/MUU//WBLL1*2/BRAMBLING & 77 & $\begin{array}{l}\text { CN079//PF70354/MUS/3/PASTOR/4/BAV92 } \\
* 2 / 5 / H A R 311\end{array}$ \\
\hline 31 & $\begin{array}{l}\text { CROC_1/AE.SQUARROSA } \\
\text { (205)//BORL95/3/PRL/SARA//TSI } \\
\text { /VEE\#5/4/FRET2/5/KINDE } \\
\end{array}$ & 78 & $\begin{array}{l}\text { CS/TH.SC//3*PVN/3/MIRLO/BUC/4/URES/JU } \\
\text { N//KAUZ/5/HUITES/6/YANAC/7/CS/TH.SC// } \\
\text { 3*PVN/3/MIRLO/BUC/4/MILAN/5/TILHI }\end{array}$ \\
\hline 32 & $\begin{array}{l}\text { KAUZ*2/MNV//KAUZ/3/MILAN/4/BAV92/5/ } \\
\text { DANPHE \#1 }\end{array}$ & 79 & 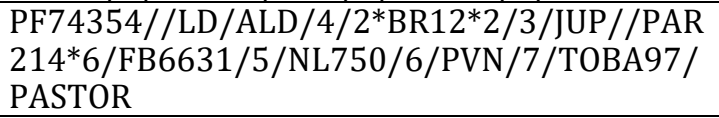 \\
\hline 33 & $\begin{array}{l}\text { THELIN/3/BABAX/LR42//BABAX/4/BABAX/ } \\
\text { LR42//BABAX/5/BOW/NKT//CBRD/3/CBRD } \\
\text { /6/FRET2*2/BRAMBLING }\end{array}$ & 80 & $\begin{array}{l}\text { BAV92//IRENA/KAUZ/3/HUITES/4/2*ROLF0 } \\
7\end{array}$ \\
\hline 34 & $\begin{array}{l}\text { WBLL1*2/KUKUNA/4/WHEAR/KUKUNA/3/C } \\
80.1 / 3 * \text { BATAVIA//2*WBLL1 }\end{array}$ & 81 & $\begin{array}{l}\text { FRET2/TUKURU//FRET2/3/MUNIA/CHTO// } \\
\text { AMSEL/4/FRET2/TUKURU//FRET2 }\end{array}$ \\
\hline 35 & $\begin{array}{l}\text { WBLL1/KUKUNA//TACUPETO } \\
\text { F2001/4/WHEAR/KUKUNA/3/C80.1/3*BATA } \\
\text { VIA//2*WBLL1 }\end{array}$ & 82 & $\begin{array}{l}\text { ATTILA*2/PBW65*2/4/BOW/NKT//CBRD/3/ } \\
\text { CBRD }\end{array}$ \\
\hline 36 & $\begin{array}{l}\text { WHEAR/KUKUNA/3/C80.1/3*BATAVIA//2* } \\
\text { WBLL1/4/QUAIU }\end{array}$ & 83 & $\begin{array}{l}\text { BAV92//IRENA/KAUZ/3/HUITES/4/FN/2*PA } \\
\text { STOR/5/BAV92//IRENA/KAUZ/3/HUITES }\end{array}$ \\
\hline 37 & $\begin{array}{l}\text { CHIBIA//PRLII/CM65531/3/FISCAL/4/ND64 } \\
3 / 2^{*} \text { WBLL1 }\end{array}$ & 84 & $\begin{array}{l}\text { NAC/TH.AC } / / 3 * \text { PVN } / 3 / \mathrm{MIRLO} / \mathrm{BUC} / 4 / 2 * \mathrm{PAS} \\
\text { TOR/5/KACHU/6/KACHU }\end{array}$ \\
\hline 38 & $\begin{array}{l}\text { DANPHE } \\
\text { \#1/3/HUW234+LR34/PRINIA//PFAU/WEAVER }\end{array}$ & 85 & $\begin{array}{l}\text { KACHU } \\
\text { \#1/6/NG8201/KAUZ/4/SHA7//PRL/VEE\# } \\
\text { 6/3/FASAN/5/MILAN/KAUZ/7/KACHU }\end{array}$ \\
\hline 39 & KACHU/BECARD//WBLL1*2/BRAMBLING & 86 & ATTILA*2/PBW65*2//MURGA \\
\hline 40 & PCAFLR/KINGBIRD \#1//KIRITATI/2*TRCH & 87 & $\begin{array}{l}\text { KBIRD//WH } \\
542 / 2 * \text { PASTOR/3/WBLL1*2/BRAMBLING }\end{array}$ \\
\hline 41 & $\begin{array}{l}\text { MUU/3/KIRITATI//ATTILA*2/PASTOR/4/MU } \\
\text { U }\end{array}$ & 88 & $\begin{array}{l}\text { KZA/5/2*WBLL1/3/STAR//KAUZ/STAR/4/B } \\
\text { AV92/RAYON }\end{array}$ \\
\hline 42 & $\begin{array}{l}\text { PRINIA/PASTOR//KIRITATI/3/PRL/2*PASTO } \\
\text { R }\end{array}$ & 89 & $\begin{array}{l}\text { WBLL1*2/KURUKU/4/BABAX/LR42//BABAX } \\
* 2 / 3 / \text { KURUKU }\end{array}$ \\
\hline 43 & $\begin{array}{l}\text { OASIS/SKAUZ//4*BCN*2/3/PASTOR/4/HEIL } \\
\text { 0/5/PAURAQ }\end{array}$ & 90 & $\begin{array}{l}\text { BAV92//IRENA/KAUZ/3/HUITES/6/ALD/CEP } \\
\text { 75630//CEP75234/PT7219/3/BUC/BJY/4/CB } \\
\text { RD/5/TNMU/PF85487 }\end{array}$ \\
\hline 44 & $\begin{array}{l}\text { ND643/2*WBLL1//ATTILA*2/PBW65/3/MU } \\
\text { NAL }\end{array}$ & 91 & $\begin{array}{l}\text { TACUPETO } \\
\text { F2001/6/CNDO/R143//ENTE/MEXI_2/3/AEG } \\
\text { ILOPS SQUARROSA } \\
\text { (TAUS)/4/WEAVER/5/PASTOR/7/ROLF07 }\end{array}$ \\
\hline 45 & $\begin{array}{l}\text { ND643/2*WBLL1/3/KIRITATI//PRL/2*PAST } \\
\text { OR/4/KIRITATI//PBW65/2*SERI.1B }\end{array}$ & 92 & $\begin{array}{l}\text { BAV92//IRENA/KAUZ/3/HUITES*2/6/TURAC } \\
\text { O/5/CHIR3/4/SIREN//ALTAR } \\
\text { 84/AE.SQUARROSA (205)/3/3*BUC }\end{array}$ \\
\hline 46 & ND643/2*TRCH//BECARD/3/BECARD & 93 & FRANCOLIN \#1/KIRITATI \\
\hline 47 & $\begin{array}{l}\text { W15.92/4/PASTOR//HXL7573/2*BAU/3/WB } \\
\text { LL1 }\end{array}$ & Morocco & e \\
\hline
\end{tabular}




\section{Disease Assessment}

Final leaf rust severities were recorded for each genotype using the modified Cobb's scale (Peterson et al., 1948). Plant reaction (infection type) was expressed in five types (Stakman et al., 1962); immune (0), resistant (R), moderately resistant (MR), moderately susceptible (MS), and susceptible (S).

The coefficient of infection (CI) was calculated by multiplying rust severity with constant values of infection type (IT). The constant values for infection types were used based on; $\mathrm{R}=0.2, \mathrm{MR}=0.4, \mathrm{MS}=0.8$ and $\mathrm{S}=1$ (Stubbs et al., 1986). The average coefficient of infection (ACI) was derived from the sum of $\mathrm{CI}$ values of each line divided by the number of locations.

The highest ACI of a candidate line is set at 100 and all other lines are adjusted accordingly. This gives the country an average relative percentage attack (CARPA). Using 0 to 9 scale previously designated as resistance index (RI) has been re-designated as a relative resistance index (RRI). From CARPA the value of RRI is calculated on 0 to 9 scale, where 0 denote most susceptible and 9 highly resistant (Aslam, 1982; Akhtar et al., 2002). The relative resistance index is calculated according to the following formula:

$$
R R I=\frac{(100-C A R P A)}{100} \times 9
$$

The desirable index and acceptable index number for rusts are as below (Aslam, 1982).

\begin{tabular}{lcc}
\hline Disease & Desirable index & Acceptable index \\
\hline $\begin{array}{l}\text { Stripe and } \\
\text { stem rust }\end{array}$ & 7 and above & 6 \\
Leaf rust & 7 and above & 6 or 5 \\
\hline
\end{tabular}

The area under the disease progress curve (AUDPC) was calculated by using the formula suggested by Pandey et al. (1989).

AUDPC $=D\left[\frac{1}{2}\left(Y_{1}+Y_{k}\right)+\left(Y_{2}+Y_{3}+\ldots \ldots+Y_{k-1}\right)\right]$

Where:

$\mathrm{D}=$ days between two consecutive records (time intervals)

$\mathrm{Y}_{1}+\mathrm{Y}_{\mathrm{k}}=$ Sum of the first and last disease scores.

$\mathrm{Y}_{2}+\mathrm{Y}_{3}+\ldots \ldots . .+\mathrm{Y}_{\mathrm{k}-1}=$ Sum of all in between disease scores.

\section{Statistical Analysis}

The mean performance of all agronomic characters of the tested genotypes was compared using the least significant difference (LSD) at $5 \%$ (Snedecor and Cochran, 1967).

\section{RESULTS}

Evaluation of Wheat Genotypes against Leaf Rust under Field Conditions

\section{Growing Season 2017/18}

Data presented in Table (2) showed that final leaf rust severity of the tested genotypes ranged from $0-70 \%$ at Menoufia and Behira from 0-90\%. All of the wheat genotypes showed resistant reaction at the two locations except 25 genotypes i.e. 3, 5, 9, 19, 20, 21, 24, 26, 38, 39, 50, 51, 53, 60, 65, 69, 72, 73, 79, 80, 85, 91, 92, 93 and Morocco (Table 2). Moreover, all of the tested wheat genotypes showed desirable/acceptable (RRI) to leaf rust ranged from 5.06 to 9.00 except the three wheat genotypes i.e. 53, 65 (each with 3.94), and Morocco (0.00) (Table 2) at the two locations during $2017 / 18$ growing season.

\section{Growing Season 2018/19}

Data in Table (3) showed that final leaf rust severity of the tested genotypes ranged from $0-90 \%$ at Menoufia and Behira from $0-100 \%$. All of the wheat genotypes showed resistant reaction at the two locations except 28 genotypes i.e. $2,5,9,18,19,20,21,23,24,26,38,39,51,53,60,62$, 65, 68, 69, 72, 73, 79, 80, 85, 91, 92, 93 and Morocco (Table 3). Moreover, all of the tested wheat genotypes showed desirable/acceptable (RRI) to leaf rust ranged from 5.21 to 9.00 except the three wheat genotypes i.e. 38 (3.32), 51 (4.74), 53 (3.79), 65 (4.74), 72 (3.32), 73 (4.74), 91 (3.32), 93 (2.37) and Morocco (0.00) (Table 3) at the two locations during 2018/19 growing season.

\section{Growing Season 2019/20}

Data in Table (4) showed that final leaf rust severity of the tested genotypes ranged from $0-60 \%$ at Menoufia and Behira from 0-70\%. All of the wheat genotypes showed resistant reaction at the two locations except 23 genotypes i.e. $3,5,9,18,19,20,21,23,24,26,32,37$, 38, $39,43,47,51,53,57,60,62,65,68,69,72,73,76,79$, 80, 83, 88, 91, 92, 93 and Morocco (Table 4). Moreover, all of the tested wheat genotypes showed desirable/acceptable (RRI) to leaf rust ranged from 5.54 to 9.00 except the three wheat genotypes i.e. $26,53,91$, 92 (each with 4.85), 93 (2.77) and Morocco (0.00) (Table 4 ) at the two locations during 2018/19 growing season. Data in Table (5) indicated all of the tested wheat genotypes were resistant to leaf rust and showed desirable/acceptable (RRI) at the two locations during the three growing seasons except 11 wheat genotypes i.e. 24, 51, 53, 65, 68, 72, 73, 91, 92, 93 and Morocco. 
Table 2: Response of 94 wheat genotypes to leaf rust along with the average coefficient of infection (ACI), country average relative percentage attack (CARPA), and relative resistance index (RRI) at Menoufia and Behira locations during 2017/18 growing season.

\begin{tabular}{|c|c|c|c|c|c|}
\hline \multirow{3}{*}{ Line } & \multicolumn{2}{|c|}{$2017 / 18$} & \multirow{3}{*}{ ACI } & \multirow{3}{*}{ CARPA } & \multirow{3}{*}{$\mathrm{RRI}^{\mathrm{b}}$} \\
\hline & \multicolumn{2}{|c|}{ Location / Final rust severity (\%) } & & & \\
\hline & Menoufia & Behira & & & \\
\hline 1 & $5 \mathrm{MR}$ & $10 \mathrm{MR}$ & 3.00 & 3.75 & 8.66 \\
\hline 2 & 0 & 0 & 0.00 & 0.00 & 9.00 \\
\hline 3 & 0 & $5 \mathrm{~S}$ & 2.50 & 3.13 & 8.72 \\
\hline 4 & $10 \mathrm{MR}$ & $5 \mathrm{MR}$ & 3.00 & 3.75 & 8.66 \\
\hline 5 & $10 \mathrm{~S}$ & $20 \mathrm{~S}$ & 15.00 & 18.75 & 7.31 \\
\hline 6 & 0 & 0 & 0.00 & 0.00 & 9.00 \\
\hline 7 & 0 & $5 \mathrm{MR}$ & 1.00 & 1.25 & 8.89 \\
\hline 8 & 0 & 0 & 0.00 & 0.00 & 9.00 \\
\hline 9 & $5 \mathrm{~S}$ & $5 \mathrm{~S}$ & 5.00 & 6.25 & 8.44 \\
\hline 10 & 0 & 0 & 0.00 & 0.00 & 9.00 \\
\hline 11 & 0 & 0 & 0.00 & 0.00 & 9.00 \\
\hline 12 & $5 \mathrm{MR}$ & $\operatorname{Tr} \mathrm{MR}$ & 1.60 & 2.00 & 8.82 \\
\hline 13 & 0 & $5 \mathrm{MR}$ & 1.00 & 1.25 & 8.89 \\
\hline 14 & 0 & 0 & 0.00 & 0.00 & 9.00 \\
\hline 15 & $10 \mathrm{MR}$ & $10 \mathrm{MR}$ & 4.00 & 5.00 & 8.55 \\
\hline 16 & 0 & 0 & 0.00 & 0.00 & 9.00 \\
\hline 17 & 0 & 0 & 0.00 & 0.00 & 9.00 \\
\hline 18 & 0 & 0 & 0.00 & 0.00 & 9.00 \\
\hline 19 & $\operatorname{Tr} \mathrm{S}$ & $10 \mathrm{~S}$ & 6.50 & 8.13 & 8.27 \\
\hline 20 & $5 \mathrm{~S}$ & $5 \mathrm{~S}$ & 5.00 & 6.25 & 8.44 \\
\hline 21 & $5 \mathrm{~S}$ & $40 \mathrm{~S}$ & 22.50 & 28.13 & 6.47 \\
\hline 22 & 0 & 0 & 0.00 & 0.00 & 9.00 \\
\hline 23 & $5 \mathrm{MR}$ & 0 & 1.00 & 1.25 & 8.89 \\
\hline 24 & $20 \mathrm{~S}$ & $5 \mathrm{~S}$ & 12.50 & 15.63 & 7.59 \\
\hline 25 & 0 & $10 \mathrm{MR}$ & 2.00 & 2.50 & 8.78 \\
\hline 26 & $30 \mathrm{MS}$ & $20 \mathrm{MS}$ & 20.00 & 25.00 & 6.75 \\
\hline 27 & 0 & 0 & 0.00 & 0.00 & 9.00 \\
\hline 28 & 0 & 0 & 0.00 & 0.00 & 9.00 \\
\hline 29 & $5 \mathrm{MR}$ & 0 & 1.00 & 1.25 & 8.89 \\
\hline 30 & 0 & 0 & 0.00 & 0.00 & 9.00 \\
\hline 31 & $20 \mathrm{MR}$ & $20 \mathrm{MR}$ & 8.00 & 10.00 & 8.10 \\
\hline 32 & 0 & 0 & 0.00 & 0.00 & 9.00 \\
\hline 33 & $5 \mathrm{R}$ & 0 & 0.50 & 0.63 & 8.94 \\
\hline 34 & 0 & 0 & 0.00 & 0.00 & 9.00 \\
\hline 35 & 0 & $10 \mathrm{R}$ & 1.00 & 1.25 & 8.89 \\
\hline 36 & 0 & 0 & 0.00 & 0.00 & 9.00 \\
\hline 37 & 0 & 0 & 0.00 & 0.00 & 9.00 \\
\hline 38 & $10 \mathrm{MS}$ & $50 \mathrm{MS}$ & 24.00 & 30.00 & 6.30 \\
\hline 39 & $5 \mathrm{~S}$ & $5 \mathrm{~S}$ & 5.00 & 6.25 & 8.44 \\
\hline 40 & 0 & 0 & 0.00 & 0.00 & 9.00 \\
\hline 41 & $20 \mathrm{R}$ & $5 \mathrm{MR}$ & 3.00 & 3.75 & 8.66 \\
\hline 42 & 0 & 0 & 0.00 & 0.00 & 9.00 \\
\hline 43 & $5 \mathrm{R}$ & $20 \mathrm{R}$ & 2.50 & 3.13 & 8.72 \\
\hline 44 & 0 & 0 & 0.00 & 0.00 & 9.00 \\
\hline 45 & 0 & 0 & 0.00 & 0.00 & 9.00 \\
\hline 46 & 0 & 0 & 0.00 & 0.00 & 9.00 \\
\hline
\end{tabular}


Table 2: Cont...

\begin{tabular}{|c|c|c|c|c|c|}
\hline 47 & 0 & 0 & 0.00 & 0.00 & 9.00 \\
\hline 48 & 0 & 0 & 0.00 & 0.00 & 9.00 \\
\hline 49 & 0 & 0 & 0.00 & 0.00 & 9.00 \\
\hline 50 & $\operatorname{Tr} \mathrm{R}$ & 0 & 0.30 & 0.38 & 8.97 \\
\hline 51 & $50 \mathrm{~S}$ & $5 \mathrm{~S}$ & 27.50 & 34.38 & 5.91 \\
\hline 52 & 0 & 0 & 0.00 & 0.00 & 9.00 \\
\hline 53 & $40 \mathrm{~S}$ & $50 \mathrm{~S}$ & 45.00 & 56.25 & 3.94 \\
\hline 54 & 0 & 0 & 0.00 & 0.00 & 9.00 \\
\hline 55 & 0 & 0 & 0.00 & 0.00 & 9.00 \\
\hline 56 & 0 & 0 & 0.00 & 0.00 & 9.00 \\
\hline 57 & 0 & 0 & 0.00 & 0.00 & 9.00 \\
\hline 58 & $5 \mathrm{MR}$ & 0 & 1.00 & 1.25 & 8.89 \\
\hline 59 & 0 & 0 & 0.00 & 0.00 & 9.00 \\
\hline 60 & $20 \mathrm{~S}$ & $5 \mathrm{~S}$ & 12.50 & 15.63 & 7.59 \\
\hline 61 & 0 & 0 & 0.00 & 0.00 & 9.00 \\
\hline 62 & $20 \mathrm{MR}$ & $20 \mathrm{MR}$ & 8.00 & 10.00 & 8.10 \\
\hline 63 & 0 & $10 \mathrm{R}$ & 1.00 & 1.25 & 8.89 \\
\hline 64 & 0 & 0 & 0.00 & 0.00 & 9.00 \\
\hline 65 & $70 \mathrm{~S}$ & $20 \mathrm{~S}$ & 45.00 & 56.25 & 3.94 \\
\hline 66 & 0 & $10 \mathrm{MR}$ & 2.00 & 2.50 & 8.78 \\
\hline 67 & 0 & 0 & 0.00 & 0.00 & 9.00 \\
\hline 68 & $5 \mathrm{R}$ & $20 \mathrm{MR}$ & 4.50 & 5.63 & 8.49 \\
\hline 69 & $30 \mathrm{MS}$ & $10 \mathrm{~S}$ & 17.00 & 21.25 & 7.09 \\
\hline 70 & 0 & 0 & 0.00 & 0.00 & 9.00 \\
\hline 71 & 0 & 0 & 0.00 & 0.00 & 9.00 \\
\hline 72 & $10 \mathrm{~S}$ & $10 \mathrm{~S}$ & 10.00 & 12.50 & 7.88 \\
\hline 73 & $5 \mathrm{MS}$ & Tr MS & 3.20 & 4.00 & 8.64 \\
\hline 74 & 0 & 0 & 0.00 & 0.00 & 9.00 \\
\hline 75 & 0 & 0 & 0.00 & 0.00 & 9.00 \\
\hline 76 & $10 \mathrm{R}$ & 0 & 1.00 & 1.25 & 8.89 \\
\hline 77 & 0 & 0 & 0.00 & 0.00 & 9.00 \\
\hline 78 & 0 & 0 & 0.00 & 0.00 & 9.00 \\
\hline 79 & $5 \mathrm{MS}$ & $10 \mathrm{~S}$ & 7.00 & 8.75 & 8.21 \\
\hline 80 & $60 \mathrm{~S}$ & $10 \mathrm{~S}$ & 35.00 & 43.75 & 5.06 \\
\hline 81 & 0 & 0 & 0.00 & 0.00 & 9.00 \\
\hline 82 & $5 \mathrm{R}$ & 0 & 0.50 & 0.63 & 8.94 \\
\hline 83 & 0 & 0 & 0.00 & 0.00 & 9.00 \\
\hline 84 & 0 & 0 & 0.00 & 0.00 & 9.00 \\
\hline 85 & $10 \mathrm{~S}$ & $5 \mathrm{~S}$ & 7.50 & 9.38 & 8.16 \\
\hline 86 & 0 & 0 & 0.00 & 0.00 & 9.00 \\
\hline 87 & $20 \mathrm{MR}$ & $10 \mathrm{MR}$ & 6.00 & 7.50 & 8.33 \\
\hline 88 & 0 & 0 & 0.00 & 0.00 & 9.00 \\
\hline 89 & 0 & $5 \mathrm{R}$ & 0.50 & 0.63 & 8.94 \\
\hline 90 & 0 & 0 & 0.00 & 0.00 & 9.00 \\
\hline 91 & $40 \mathrm{~S}$ & $10 \mathrm{~S}$ & 25.00 & 31.25 & 6.19 \\
\hline 92 & $5 \mathrm{~S}$ & $30 \mathrm{~S}$ & 17.50 & 21.88 & 7.03 \\
\hline 93 & $20 \mathrm{~S}$ & $50 \mathrm{~S}$ & 35.00 & 43.75 & 5.06 \\
\hline Morocco & $70 \mathrm{~S}$ & $90 \mathrm{~S}$ & 80.00 & 100.00 & 0.00 \\
\hline LSD at $5 \%$ & & & & & 0.761 \\
\hline
\end{tabular}

a Final rust severity includes two components: disease severity based on modified Cobb's scale (Peterson et al., 1948), where $\mathrm{Tr}=$ less than $5 \%$ and $5=5 \%$ up to $100=100 \%$, and host response based on scale described by Stakman $e t$ al. (1962), where $\mathrm{R}=$ resistant, $\mathrm{MR}=$ moderately resistant, $\mathrm{MS}=$ moderately susceptible and $\mathrm{S}=$ susceptible. b RRI= Relative resistance index (above 5 is acceptable; means the variety is resistant to rusts (Aslam, 1982). 
Table 3: Response of 94 wheat genotypes to leaf rust along with the average coefficient of infection (ACI), country average relative percentage attack (CARPA), and relative resistance index (RRI) at Menoufia and Behira locations during 2018/19 growing season.

\begin{tabular}{|c|c|c|c|c|c|}
\hline \multirow{3}{*}{ Line } & \multicolumn{2}{|c|}{$2018 / 19$} & \multirow{3}{*}{ ACI } & \multirow{3}{*}{ CARPA } & \multirow{3}{*}{ RRI } \\
\hline & \multicolumn{2}{|c|}{ Location / Final rust severity (\%) } & & & \\
\hline & Menoufia & Behira & & & \\
\hline 1 & 0 & 0 & 0.00 & 0.00 & 9.00 \\
\hline 2 & 0 & 0 & 0.00 & 0.00 & 9.00 \\
\hline 3 & $20 \mathrm{~S}$ & $30 \mathrm{~S}$ & 25.00 & 26.32 & 6.63 \\
\hline 4 & 0 & $10 \mathrm{MR}$ & 2.00 & 2.11 & 8.81 \\
\hline 5 & $\operatorname{Tr} \mathrm{S}$ & $50 \mathrm{~S}$ & 26.50 & 27.89 & 6.49 \\
\hline 6 & $5 \mathrm{MR}$ & $10 \mathrm{MR}$ & 3.00 & 3.16 & 8.72 \\
\hline 7 & 0 & 0 & 0.00 & 0.00 & 9.00 \\
\hline 8 & 0 & 0 & 0.00 & 0.00 & 9.00 \\
\hline 9 & $10 \mathrm{MS}$ & $10 \mathrm{MS}$ & 8.00 & 8.42 & 8.24 \\
\hline 10 & $5 \mathrm{R}$ & $10 \mathrm{MR}$ & 2.50 & 2.63 & 8.76 \\
\hline 11 & 0 & 0 & 0.00 & 0.00 & 9.00 \\
\hline 12 & 0 & 0 & 0.00 & 0.00 & 9.00 \\
\hline 13 & $20 \mathrm{MR}$ & $10 \mathrm{MR}$ & 6.00 & 6.32 & 8.43 \\
\hline 14 & $10 \mathrm{R}$ & $20 \mathrm{MR}$ & 5.00 & 5.26 & 8.53 \\
\hline 15 & 0 & 0 & 0.00 & 0.00 & 9.00 \\
\hline 16 & 0 & 0 & 0.00 & 0.00 & 9.00 \\
\hline 17 & 0 & 0 & 0.00 & 0.00 & 9.00 \\
\hline 18 & $20 \mathrm{MR}$ & $10 \mathrm{MS}$ & 8.00 & 8.42 & 8.24 \\
\hline 19 & $30 \mathrm{~S}$ & $20 \mathrm{~S}$ & 25.00 & 26.32 & 6.63 \\
\hline 20 & $10 \mathrm{~S}$ & $30 \mathrm{~S}$ & 20.00 & 21.05 & 7.11 \\
\hline 21 & $20 \mathrm{~S}$ & $5 \mathrm{~S}$ & 12.50 & 13.16 & 7.82 \\
\hline 22 & $20 \mathrm{R}$ & $10 \mathrm{R}$ & 3.00 & 3.16 & 8.72 \\
\hline 23 & $10 \mathrm{~S}$ & $\operatorname{Tr} S$ & 6.50 & 6.84 & 8.38 \\
\hline 24 & $50 \mathrm{~S}$ & $40 \mathrm{~S}$ & 45.00 & 47.37 & 4.74 \\
\hline 25 & $20 \mathrm{MR}$ & $\operatorname{Tr} M R$ & 4.60 & 4.84 & 8.56 \\
\hline 26 & $20 \mathrm{~S}$ & $60 \mathrm{~S}$ & 40.00 & 42.11 & 5.21 \\
\hline 27 & 0 & 0 & 0.00 & 0.00 & 9.00 \\
\hline 28 & 0 & 0 & 0.00 & 0.00 & 9.00 \\
\hline 29 & 0 & 0 & 0.00 & 0.00 & 9.00 \\
\hline 30 & 0 & 0 & 0.00 & 0.00 & 9.00 \\
\hline 31 & 0 & 0 & 0.00 & 0.00 & 9.00 \\
\hline 32 & 0 & 0 & 0.00 & 0.00 & 9.00 \\
\hline 33 & 0 & 0 & 0.00 & 0.00 & 9.00 \\
\hline 34 & 0 & 0 & 0.00 & 0.00 & 9.00 \\
\hline 35 & 0 & 0 & 0.00 & 0.00 & 9.00 \\
\hline 36 & 0 & 0 & 0.00 & 0.00 & 9.00 \\
\hline 37 & 0 & 0 & 0.00 & 0.00 & 9.00 \\
\hline 38 & $60 \mathrm{~S}$ & $60 \mathrm{~S}$ & 60.00 & 63.16 & 3.32 \\
\hline 39 & $20 \mathrm{~S}$ & $40 \mathrm{~S}$ & 30.00 & 31.58 & 6.16 \\
\hline 40 & $5 \mathrm{R}$ & $\operatorname{Tr} \mathrm{R}$ & 0.80 & 0.84 & 8.92 \\
\hline 41 & 0 & 0 & 0.00 & 0.00 & 9.00 \\
\hline 42 & $5 \mathrm{MR}$ & $10 \mathrm{MR}$ & 3.00 & 3.16 & 8.72 \\
\hline 43 & 0 & 0 & 0.00 & 0.00 & 9.00 \\
\hline 44 & 0 & 0 & 0.00 & 0.00 & 9.00 \\
\hline 45 & 0 & 0 & 0.00 & 0.00 & 9.00 \\
\hline 46 & 0 & 0 & 0.00 & 0.00 & 9.00 \\
\hline
\end{tabular}


Table 3: Cont...

\begin{tabular}{|c|c|c|c|c|c|}
\hline 47 & 0 & 0 & 0.00 & 0.00 & 9.00 \\
\hline 48 & 0 & 0 & 0.00 & 0.00 & 9.00 \\
\hline 49 & 0 & 0 & 0.00 & 0.00 & 9.00 \\
\hline 50 & $20 \mathrm{R}$ & $10 \mathrm{R}$ & 3.00 & 3.16 & 8.72 \\
\hline 51 & $30 \mathrm{~S}$ & $60 \mathrm{~S}$ & 45.00 & 47.37 & 4.74 \\
\hline 52 & 0 & 0 & 0.00 & 0.00 & 9.00 \\
\hline 53 & $70 \mathrm{~S}$ & $40 \mathrm{~S}$ & 55.00 & 57.89 & 3.79 \\
\hline 54 & 0 & 0 & 0.00 & 0.00 & 9.00 \\
\hline 55 & 0 & 0 & 0.00 & 0.00 & 9.00 \\
\hline 56 & 0 & 0 & 0.00 & 0.00 & 9.00 \\
\hline 57 & 0 & 0 & 0.00 & 0.00 & 9.00 \\
\hline 58 & 0 & 0 & 0.00 & 0.00 & 9.00 \\
\hline 59 & 0 & 0 & 0.00 & 0.00 & 9.00 \\
\hline 60 & $10 \mathrm{MR}$ & $20 \mathrm{~S}$ & 12.00 & 12.63 & 7.86 \\
\hline 61 & 5S & 0 & 2.50 & 2.63 & 8.76 \\
\hline 62 & $30 \mathrm{MS}$ & $30 \mathrm{MS}$ & 24.00 & 25.26 & 6.73 \\
\hline 63 & 0 & 0 & 0.00 & 0.00 & 9.00 \\
\hline 64 & 0 & 0 & 0.00 & 0.00 & 9.00 \\
\hline 65 & $50 \mathrm{~S}$ & $40 \mathrm{~S}$ & 45.00 & 47.37 & 4.74 \\
\hline 66 & 0 & 0 & 0.00 & 0.00 & 9.00 \\
\hline 67 & 0 & 0 & 0.00 & 0.00 & 9.00 \\
\hline 68 & $20 \mathrm{~S}$ & $70 \mathrm{~S}$ & 45.00 & 47.37 & 4.74 \\
\hline 69 & $60 \mathrm{~S}$ & $20 \mathrm{~S}$ & 40.00 & 42.11 & 5.21 \\
\hline 70 & $10 \mathrm{R}$ & $30 \mathrm{MR}$ & 7.00 & 7.37 & 8.34 \\
\hline 71 & $5 \mathrm{MR}$ & $10 \mathrm{MR}$ & 3.00 & 3.16 & 8.72 \\
\hline 72 & $70 \mathrm{~S}$ & $50 \mathrm{~S}$ & 60.00 & 63.16 & 3.32 \\
\hline 73 & $40 \mathrm{~S}$ & $50 \mathrm{~S}$ & 45.00 & 47.37 & 4.74 \\
\hline 74 & 0 & 0 & 0.00 & 0.00 & 9.00 \\
\hline 75 & 0 & 0 & 0.00 & 0.00 & 9.00 \\
\hline 76 & 0 & 0 & 0.00 & 0.00 & 9.00 \\
\hline 77 & 0 & 0 & 0.00 & 0.00 & 9.00 \\
\hline 78 & 0 & 0 & 0.00 & 0.00 & 9.00 \\
\hline 79 & $30 \mathrm{~S}$ & $60 \mathrm{~S}$ & 45.00 & 47.37 & 4.74 \\
\hline 80 & $30 \mathrm{~S}$ & $30 \mathrm{~S}$ & 30.00 & 31.58 & 6.16 \\
\hline 81 & 0 & 0 & 0.00 & 0.00 & 9.00 \\
\hline 82 & 0 & 0 & 0.00 & 0.00 & 9.00 \\
\hline 83 & 0 & 0 & 0.00 & 0.00 & 9.00 \\
\hline 84 & 0 & 0 & 0.00 & 0.00 & 9.00 \\
\hline 85 & $40 \mathrm{~S}$ & $10 \mathrm{~S}$ & 25.00 & 26.32 & 6.63 \\
\hline 86 & 0 & 0 & 0.00 & 0.00 & 9.00 \\
\hline 87 & 0 & 0 & 0.00 & 0.00 & 9.00 \\
\hline 88 & 0 & 0 & 0.00 & 0.00 & 9.00 \\
\hline 89 & 0 & 0 & 0.00 & 0.00 & 9.00 \\
\hline 90 & 0 & 0 & 0.00 & 0.00 & 9.00 \\
\hline 91 & $70 \mathrm{~S}$ & $50 \mathrm{~S}$ & 60.00 & 63.16 & 3.32 \\
\hline 92 & $30 \mathrm{~S}$ & $10 \mathrm{~S}$ & 20.00 & 21.05 & 7.11 \\
\hline 93 & $60 \mathrm{~S}$ & $80 \mathrm{~S}$ & 70.00 & 73.68 & 2.37 \\
\hline Morocco & $90 \mathrm{~S}$ & $100 \mathrm{~S}$ & 95.00 & 100.00 & 0.00 \\
\hline LSD at $5 \%$ & & & & & 0.965 \\
\hline
\end{tabular}

a Final rust severity includes two components: disease severity based on modified Cobb's scale (Peterson et al., 1948), where $\mathrm{Tr}=$ less than $5 \%$ and $5=5 \%$ up to $100=100 \%$, and host response based on scale described by Stakman et al. (1962), where $\mathrm{R}=$ resistant, $\mathrm{MR}=$ moderately resistant, $\mathrm{MS}=$ moderately susceptible and $\mathrm{S}=$ susceptible. b RRI= Relative resistance index (above 5 is acceptable; means the variety is resistant to rusts (Aslam, 1982). 
Table 4: Response of 94 wheat genotypes to leaf rust along with the average coefficient of infection (ACI), country average relative percentage attack (CARPA), and relative resistance index (RRI) at Menoufia and Behira locations during 2019/20 growing season.

\begin{tabular}{|c|c|c|c|c|c|}
\hline \multirow{3}{*}{ Line } & \multicolumn{2}{|c|}{$2019 / 20$} & \multirow{3}{*}{ ACI } & \multirow{3}{*}{ CARPA } & \multirow{3}{*}{$\mathrm{RRI}^{\mathrm{b}}$} \\
\hline & \multicolumn{2}{|c|}{ Location / Final rust severity (\%) ${ }^{a}$} & & & \\
\hline & Menoufia & Behira & & & \\
\hline 1 & 0 & 0 & 0.00 & 0.00 & 9.00 \\
\hline 2 & 0 & 0 & 0.00 & 0.00 & 9.00 \\
\hline 3 & $5 \mathrm{~S}$ & $10 \mathrm{~S}$ & 7.50 & 11.54 & 7.96 \\
\hline 4 & 0 & 0 & 0.00 & 0.00 & 9.00 \\
\hline 5 & $\operatorname{Tr} S$ & $10 \mathrm{~S}$ & 6.50 & 10.00 & 8.10 \\
\hline 6 & 0 & 0 & 0.00 & 0.00 & 9.00 \\
\hline 7 & 0 & 0 & 0.00 & 0.00 & 9.00 \\
\hline 8 & 0 & 0 & 0.00 & 0.00 & 9.00 \\
\hline 9 & $5 \mathrm{MS}$ & $5 \mathrm{MS}$ & 4.00 & 6.15 & 8.45 \\
\hline 10 & 0 & 0 & 0.00 & 0.00 & 9.00 \\
\hline 11 & 0 & 0 & 0.00 & 0.00 & 9.00 \\
\hline 12 & 0 & 0 & 0.00 & 0.00 & 9.00 \\
\hline 13 & 0 & 0 & 0.00 & 0.00 & 9.00 \\
\hline 14 & 0 & 0 & 0.00 & 0.00 & 9.00 \\
\hline 15 & 0 & 0 & 0.00 & 0.00 & 9.00 \\
\hline 16 & 0 & 0 & 0.00 & 0.00 & 9.00 \\
\hline 17 & 0 & 0 & 0.00 & 0.00 & 9.00 \\
\hline 18 & $5 \mathrm{~S}$ & $5 \mathrm{~S}$ & 5.00 & 7.69 & 8.31 \\
\hline 19 & $10 \mathrm{~S}$ & $5 \mathrm{~S}$ & 7.50 & 11.54 & 7.96 \\
\hline 20 & $5 \mathrm{~S}$ & $10 \mathrm{~S}$ & 7.50 & 11.54 & 7.96 \\
\hline 21 & $10 \mathrm{~S}$ & $10 \mathrm{~S}$ & 10.00 & 15.38 & 7.62 \\
\hline 22 & 0 & 0 & 0.00 & 0.00 & 9.00 \\
\hline 23 & $5 \mathrm{~S}$ & $5 \mathrm{~S}$ & 5.00 & 7.69 & 8.31 \\
\hline 24 & $20 \mathrm{~S}$ & $10 \mathrm{~S}$ & 15.00 & 23.08 & 6.92 \\
\hline 25 & 0 & 0 & 0.00 & 0.00 & 9.00 \\
\hline 26 & $10 \mathrm{~S}$ & $50 \mathrm{~S}$ & 30.00 & 46.15 & 4.85 \\
\hline 27 & 0 & 0 & 0.00 & 0.00 & 9.00 \\
\hline 28 & 0 & 0 & 0.00 & 0.00 & 9.00 \\
\hline 29 & 0 & 0 & 0.00 & 0.00 & 9.00 \\
\hline 30 & 0 & 0 & 0.00 & 0.00 & 9.00 \\
\hline 31 & 0 & 0 & 0.00 & 0.00 & 9.00 \\
\hline 32 & $\operatorname{Tr} \mathrm{S}$ & $\operatorname{Tr} \mathrm{S}$ & 3.00 & 4.62 & 8.58 \\
\hline 33 & 0 & 0 & 0.00 & 0.00 & 9.00 \\
\hline 34 & 0 & 0 & 0.00 & 0.00 & 9.00 \\
\hline 35 & 0 & 0 & 0.00 & 0.00 & 9.00 \\
\hline 36 & 0 & 0 & 0.00 & 0.00 & 9.00 \\
\hline 37 & $\operatorname{Tr} S$ & $5 \mathrm{~S}$ & 4.00 & 6.15 & 8.45 \\
\hline 38 & $20 \mathrm{~S}$ & $5 \mathrm{~S}$ & 12.50 & 19.23 & 7.27 \\
\hline 39 & $\operatorname{Tr} \mathrm{S}$ & $5 \mathrm{~S}$ & 4.00 & 6.15 & 8.45 \\
\hline 40 & 0 & 0 & 0.00 & 0.00 & 9.00 \\
\hline 41 & 0 & 0 & 0.00 & 0.00 & 9.00 \\
\hline 42 & 0 & 0 & 0.00 & 0.00 & 9.00 \\
\hline 43 & $\operatorname{Tr} \mathrm{S}$ & $5 \mathrm{~S}$ & 4.00 & 6.15 & 8.45 \\
\hline 44 & 0 & 0 & 0.00 & 0.00 & 9.00 \\
\hline 45 & 0 & 0 & 0.00 & 0.00 & 9.00 \\
\hline 46 & $\operatorname{Tr} S$ & $\operatorname{Tr} S$ & 3.00 & 4.62 & 8.58 \\
\hline
\end{tabular}


Table 4: Cont...

\begin{tabular}{|c|c|c|c|c|c|}
\hline 47 & $\operatorname{Tr} \mathrm{S}$ & $\operatorname{Tr} \mathrm{S}$ & 3.00 & 4.62 & 8.58 \\
\hline 48 & 0 & 0 & 0.00 & 0.00 & 9.00 \\
\hline 49 & 0 & 0 & 0.00 & 0.00 & 9.00 \\
\hline 50 & 0 & 0 & 0.00 & 0.00 & 9.00 \\
\hline 51 & $10 \mathrm{~S}$ & $5 \mathrm{~S}$ & 7.50 & 11.54 & 7.96 \\
\hline 52 & 0 & 0 & 0.00 & 0.00 & 9.00 \\
\hline 53 & $30 \mathrm{~S}$ & $30 \mathrm{~S}$ & 30.00 & 46.15 & 4.85 \\
\hline 54 & 0 & 0 & 0.00 & 0.00 & 9.00 \\
\hline 55 & 0 & 0 & 0.00 & 0.00 & 9.00 \\
\hline 56 & 0 & 0 & 0.00 & 0.00 & 9.00 \\
\hline 57 & $\operatorname{Tr} \mathrm{S}$ & $\operatorname{Tr} \mathrm{S}$ & 3.00 & 4.62 & 8.58 \\
\hline 58 & 0 & 0 & 0.00 & 0.00 & 9.00 \\
\hline 59 & 0 & 0 & 0.00 & 0.00 & 9.00 \\
\hline 60 & $\operatorname{Tr} \mathrm{S}$ & $5 \mathrm{~S}$ & 4.00 & 6.15 & 8.45 \\
\hline 61 & $5 S$ & 0 & 2.50 & 3.85 & 8.65 \\
\hline 62 & $5 \mathrm{~S}$ & $20 \mathrm{~S}$ & 12.50 & 19.23 & 7.27 \\
\hline 63 & 0 & 0 & 0.00 & 0.00 & 9.00 \\
\hline 64 & 0 & 0 & 0.00 & 0.00 & 9.00 \\
\hline 65 & $10 \mathrm{~S}$ & $30 \mathrm{~S}$ & 20.00 & 30.77 & 6.23 \\
\hline 66 & 0 & 0 & 0.00 & 0.00 & 9.00 \\
\hline 67 & 0 & 0 & 0.00 & 0.00 & 9.00 \\
\hline 68 & $5 \mathrm{~S}$ & $10 \mathrm{~S}$ & 7.50 & 11.54 & 7.96 \\
\hline 69 & $20 \mathrm{~S}$ & $30 \mathrm{~S}$ & 25.00 & 38.46 & 5.54 \\
\hline 70 & 0 & 0 & 0.00 & 0.00 & 9.00 \\
\hline 71 & 0 & 0 & 0.00 & 0.00 & 9.00 \\
\hline 72 & $20 \mathrm{~S}$ & $20 \mathrm{~S}$ & 20.00 & 30.77 & 6.23 \\
\hline 73 & $5 \mathrm{~S}$ & $30 \mathrm{~S}$ & 17.50 & 26.92 & 6.58 \\
\hline 74 & 0 & 0 & 0.00 & 0.00 & 9.00 \\
\hline 75 & 0 & 0 & 0.00 & 0.00 & 9.00 \\
\hline 76 & $\operatorname{Tr} \mathrm{S}$ & $\operatorname{Tr} \mathrm{S}$ & 3.00 & 4.62 & 8.58 \\
\hline 77 & 0 & 0 & 0.00 & 0.00 & 9.00 \\
\hline 78 & 0 & 0 & 0.00 & 0.00 & 9.00 \\
\hline 79 & $10 \mathrm{~S}$ & $20 \mathrm{~S}$ & 15.00 & 23.08 & 6.92 \\
\hline 80 & $5 \mathrm{~S}$ & $20 \mathrm{~S}$ & 12.50 & 19.23 & 7.27 \\
\hline 81 & 0 & 0 & 0.00 & 0.00 & 9.00 \\
\hline 82 & 0 & 0 & 0.00 & 0.00 & 9.00 \\
\hline 83 & $\operatorname{Tr} \mathrm{S}$ & $\operatorname{Tr} \mathrm{S}$ & 3.00 & 4.62 & 8.58 \\
\hline 84 & 0 & 0 & 0.00 & 0.00 & 9.00 \\
\hline 85 & $5 \mathrm{~S}$ & $10 \mathrm{~S}$ & 7.50 & 11.54 & 7.96 \\
\hline 86 & 0 & 0 & 0.00 & 0.00 & 9.00 \\
\hline 87 & 0 & 0 & 0.00 & 0.00 & 9.00 \\
\hline 88 & $\operatorname{Tr} \mathrm{S}$ & $5 \mathrm{~S}$ & 4.00 & 6.15 & 8.45 \\
\hline 89 & 0 & 0 & 0.00 & 0.00 & 9.00 \\
\hline 90 & 0 & 0 & 0.00 & 0.00 & 9.00 \\
\hline 91 & $30 \mathrm{~S}$ & $30 \mathrm{~S}$ & 30.00 & 46.15 & 4.85 \\
\hline 92 & $20 \mathrm{~S}$ & $40 \mathrm{~S}$ & 30.00 & 46.15 & 4.85 \\
\hline 93 & $50 \mathrm{~S}$ & $40 \mathrm{~S}$ & 45.00 & 69.23 & 2.77 \\
\hline Morocco & $60 \mathrm{~S}$ & $70 \mathrm{~S}$ & 65.00 & 100.00 & 0.00 \\
\hline LSD at $5 \%$ & & & & & 0.679 \\
\hline
\end{tabular}

a Final rust severity includes two components: disease severity based on modified Cobb's scale (Peterson et al., 1948), where $\mathrm{Tr}=$ less than $5 \%$ and $5=5 \%$ up to $100=100 \%$, and host response based on scale described by Stakman $e t$ al. (1962), where $\mathrm{R}=$ resistant, $\mathrm{MR}=$ moderately resistant, $\mathrm{MS}=$ moderately susceptible and $\mathrm{S}=$ susceptible. b RRI= Relative resistance index (above 5 is acceptable; means the variety is resistant to rusts (Aslam, 1982). 
Table 5: Resistant wheat genotypes with desirable and acceptable relative resistance index (RRI) to leaf rust disease at Menoufia and Behira locations during 2017/18,2018/19, and 2019/20 growing seasons.

\begin{tabular}{|c|c|c|c|}
\hline \multirow{2}{*}{ Line } & \multicolumn{3}{|c|}{ Season / RRI } \\
\hline & $2017 / 18$ & $2018 / 19$ & $2019 / 20$ \\
\hline 1 & 8.66 & 9.00 & 9.00 \\
\hline 2 & 9.00 & 9.00 & 9.00 \\
\hline 3 & 8.72 & 6.63 & 7.96 \\
\hline 4 & 8.66 & 8.81 & 9.00 \\
\hline 5 & 7.31 & 6.49 & 8.10 \\
\hline 6 & 9.00 & 8.72 & 9.00 \\
\hline 7 & 8.89 & 9.00 & 9.00 \\
\hline 8 & 9.00 & 9.00 & 9.00 \\
\hline 9 & 8.44 & 8.24 & 8.45 \\
\hline 10 & 9.00 & 8.76 & 9.00 \\
\hline 11 & 9.00 & 9.00 & 9.00 \\
\hline 12 & 8.82 & 9.00 & 9.00 \\
\hline 13 & 8.89 & 8.43 & 9.00 \\
\hline 14 & 9.00 & 8.53 & 9.00 \\
\hline 15 & 8.55 & 9.00 & 9.00 \\
\hline 16 & 9.00 & 9.00 & 9.00 \\
\hline 17 & 9.00 & 9.00 & 9.00 \\
\hline 18 & 9.00 & 8.24 & 8.31 \\
\hline 19 & 8.27 & 6.63 & 7.96 \\
\hline 20 & 8.44 & 7.11 & 7.96 \\
\hline 21 & 6.47 & 7.82 & 7.62 \\
\hline 22 & 9.00 & 8.72 & 9.00 \\
\hline 23 & 8.89 & 8.38 & 8.31 \\
\hline 25 & 8.78 & 8.56 & 9.00 \\
\hline 26 & 6.75 & 5.21 & 4.85 \\
\hline 27 & 9.00 & 9.00 & 9.00 \\
\hline 28 & 9.00 & 9.00 & 9.00 \\
\hline 29 & 8.89 & 9.00 & 9.00 \\
\hline 30 & 9.00 & 9.00 & 9.00 \\
\hline 31 & 8.10 & 9.00 & 9.00 \\
\hline 32 & 9.00 & 9.00 & 8.58 \\
\hline 33 & 8.94 & 9.00 & 9.00 \\
\hline 34 & 9.00 & 9.00 & 9.00 \\
\hline 35 & 8.89 & 9.00 & 9.00 \\
\hline 36 & 9.00 & 9.00 & 9.00 \\
\hline 37 & 9.00 & 9.00 & 8.45 \\
\hline 38 & 6.30 & 3.32 & 7.27 \\
\hline 39 & 8.44 & 6.16 & 8.45 \\
\hline 40 & 9.00 & 8.92 & 9.00 \\
\hline 41 & 8.66 & 9.00 & 9.00 \\
\hline 42 & 9.00 & 8.72 & 9.00 \\
\hline 43 & 8.72 & 9.00 & 8.45 \\
\hline
\end{tabular}


Table 5: Cont...

\begin{tabular}{|c|c|c|c|}
\hline 44 & 9.00 & 9.00 & 9.00 \\
\hline 45 & 9.00 & 9.00 & 9.00 \\
\hline 46 & 9.00 & 9.00 & 8.58 \\
\hline 47 & 9.00 & 9.00 & 8.58 \\
\hline 48 & 9.00 & 9.00 & 9.00 \\
\hline 49 & 9.00 & 9.00 & 9.00 \\
\hline 50 & 8.97 & 8.72 & 9.00 \\
\hline 52 & 9.00 & 9.00 & 9.00 \\
\hline 54 & 9.00 & 9.00 & 9.00 \\
\hline 55 & 9.00 & 9.00 & 9.00 \\
\hline 56 & 9.00 & 9.00 & 9.00 \\
\hline 57 & 9.00 & 9.00 & 8.58 \\
\hline 58 & 8.89 & 9.00 & 9.00 \\
\hline 59 & 9.00 & 9.00 & 9.00 \\
\hline 60 & 7.59 & 7.86 & 8.45 \\
\hline 61 & 9.00 & 8.76 & 8.65 \\
\hline 62 & 8.10 & 6.73 & 7.27 \\
\hline 63 & 8.89 & 9.00 & 9.00 \\
\hline 64 & 9.00 & 9.00 & 9.00 \\
\hline 66 & 8.78 & 9.00 & 9.00 \\
\hline 67 & 9.00 & 9.00 & 9.00 \\
\hline 69 & 7.09 & 5.21 & 5.54 \\
\hline 70 & 9.00 & 8.34 & 9.00 \\
\hline 71 & 9.00 & 8.72 & 9.00 \\
\hline 74 & 9.00 & 9.00 & 9.00 \\
\hline 75 & 9.00 & 9.00 & 9.00 \\
\hline 76 & 8.89 & 9.00 & 8.58 \\
\hline 77 & 9.00 & 9.00 & 9.00 \\
\hline 78 & 9.00 & 9.00 & 9.00 \\
\hline 79 & 8.21 & 4.74 & 6.92 \\
\hline 80 & 5.06 & 6.16 & 7.27 \\
\hline 81 & 9.00 & 9.00 & 9.00 \\
\hline 82 & 8.94 & 9.00 & 9.00 \\
\hline 83 & 9.00 & 9.00 & 8.58 \\
\hline 84 & 9.00 & 9.00 & 9.00 \\
\hline 85 & 8.16 & 6.63 & 7.96 \\
\hline 86 & 9.00 & 9.00 & 9.00 \\
\hline 87 & 8.33 & 9.00 & 9.00 \\
\hline 88 & 9.00 & 9.00 & 8.45 \\
\hline 89 & 8.94 & 9.00 & 9.00 \\
\hline 90 & 9.00 & 9.00 & 9.00 \\
\hline
\end{tabular}

\section{Area under Disease Progress Curve (AUDPC)}

The AUDPC values during the 2016/17 and 2017/18 growing season ranged from 0.0 to 1120.0 at the two locations. While during the 2018/19 growing seasons,
AUDPC values ranged from 0.0 to 1225.0 . Moreover, during the three growing seasons of the study at the two locations, AUDPC values ranged from 0.0 to 974.17 (Table 6). 
Table 6: Area under disease progress curve (AUDPC) of 94 wheat genotypes to leaf rust at Menoufia and Behira locations during 2017/18, 2018/19, and 2019/20 growing seasons.

\begin{tabular}{|c|c|c|c|c|c|c|c|}
\hline \multirow{3}{*}{ Line } & \multicolumn{6}{|c|}{ Location / Season / AUDPC } & \multirow{3}{*}{ Mean } \\
\hline & \multicolumn{3}{|c|}{ Menoufia } & \multicolumn{3}{|c|}{ Behira } & \\
\hline & $2017 / 18$ & $2018 / 19$ & $2019 / 20$ & $2017 / 18$ & $2018 / 19$ & $2019 / 20$ & \\
\hline 1 & 49.0 & 0.0 & 0.0 & 80.5 & 0.0 & 0.0 & 21.58 \\
\hline 2 & 0.0 & 0.0 & 0.0 & 0.0 & 0.0 & 0.0 & 0.00 \\
\hline 3 & 0.0 & 157.5 & 49.0 & 49.0 & 252.0 & 80.5 & 98.00 \\
\hline 4 & 80.5 & 0.0 & 0.0 & 49.0 & 80.5 & 0.0 & 35.00 \\
\hline 5 & 80.5 & 42.0 & 42.0 & 157.5 & 455.0 & 80.5 & 142.92 \\
\hline 6 & 0.0 & 49.0 & 0.0 & 0.0 & 80.5 & 0.0 & 21.58 \\
\hline 7 & 0.0 & 0.0 & 0.0 & 49.0 & 0.0 & 0.0 & 8.17 \\
\hline 8 & 0.0 & 0.0 & 0.0 & 0.0 & 0.0 & 0.0 & 0.00 \\
\hline 9 & 49.0 & 80.5 & 49.0 & 49.0 & 80.5 & 49.0 & 59.50 \\
\hline 10 & 0.0 & 5.0 & 0.0 & 0.0 & 80.5 & 0.0 & 14.25 \\
\hline 11 & 0.0 & 0.0 & 0.0 & 0.0 & 0.0 & 0.0 & 0.00 \\
\hline 12 & 49.0 & 0.0 & 0.0 & $42 \mathrm{M}$ & 0.0 & 0.0 & 8.17 \\
\hline 13 & 0.0 & 157.5 & 0.0 & 49.0 & 80.5 & 0.0 & 47.83 \\
\hline 14 & 0.0 & 80.5 & 0.0 & 0.0 & 157.5 & 0.0 & 39.67 \\
\hline 15 & 80.5 & 0.0 & 0.0 & 80.5 & 0.0 & 0.0 & 26.83 \\
\hline 16 & 0.0 & 0.0 & 0.0 & 0.0 & 0.0 & 0.0 & 0.00 \\
\hline 17 & 0.0 & 0.0 & 0.0 & 0.0 & 0.0 & 0.0 & 0.00 \\
\hline 18 & 0.0 & 157.5 & 49.0 & 0.0 & 80.5 & 49.0 & 56.00 \\
\hline 19 & 42.0 & 252.0 & 80.5 & 80.5 & 157.5 & 49.0 & 110.25 \\
\hline 20 & 49.0 & 80.5 & 49.0 & 49.0 & 252.0 & 80.5 & 93.33 \\
\hline 21 & 49.0 & 157.5 & 80.5 & 385.0 & 49.0 & 80.5 & 133.58 \\
\hline 22 & 0.0 & 157.5 & 0.0 & 0.0 & 80.5 & 0.0 & 39.67 \\
\hline 23 & 49.0 & 80.5 & 49.0 & 0.0 & 42.0 & 49.0 & 44.92 \\
\hline 24 & 157.5 & 455.0 & 157.5 & 49.0 & 385.0 & 80.5 & 214.08 \\
\hline 25 & 0.0 & 157.5 & 0.0 & 80.5 & $42 \mathrm{M}$ & 0.0 & 39.67 \\
\hline 26 & 252.0 & 157.5 & 80.5 & 157.5 & 700.0 & 455.0 & 300.42 \\
\hline 27 & 0.0 & 0.0 & 0.0 & 0.0 & 0.0 & 0.0 & 0.00 \\
\hline 28 & 0.0 & 0.0 & 0.0 & 0.0 & 0.0 & 0.0 & 0.00 \\
\hline 29 & 49.0 & 0.0 & 0.0 & 0.0 & 0.0 & 0.0 & 8.17 \\
\hline 30 & 0.0 & 0.0 & 0.0 & 0.0 & 0.0 & 0.0 & 0.00 \\
\hline 31 & 157.5 & 0.0 & 0.0 & 157.5 & 0.0 & 0.0 & 52.50 \\
\hline 32 & 0.0 & 0.0 & 42.0 & 0.0 & 0.0 & 42.0 & 14.00 \\
\hline 33 & 5.0 & 0.0 & 0.0 & 0.0 & 0.0 & 0.0 & 0.83 \\
\hline 34 & 0.0 & 0.0 & 0.0 & 0.0 & 0.0 & 0.0 & 0.00 \\
\hline 35 & 0.0 & 0.0 & 0.0 & 80.5 & 0.0 & 0.0 & 13.42 \\
\hline 36 & 0.0 & 0.0 & 0.0 & 0.0 & 0.0 & 0.0 & 0.00 \\
\hline 37 & 0.0 & 0.0 & 42.0 & 0.0 & 0.0 & 49.0 & 15.17 \\
\hline 38 & 80.5 & 700.0 & 157.5 & 455.0 & 700.0 & 49.0 & 357.00 \\
\hline 39 & 49.0 & 157.5 & 42.0 & 49.0 & 385.0 & 49.0 & 121.92 \\
\hline 40 & 0.0 & 5.0 & 0.0 & 0.0 & 42.0 & 0.0 & 7.83 \\
\hline 41 & 157.5 & 0.0 & 0.0 & 49.0 & 0.0 & 0.0 & 34.42 \\
\hline 42 & 0.0 & 49.0 & 0.0 & 0.0 & 80.5 & 0.0 & 21.58 \\
\hline 43 & 5.0 & 0.0 & 42.0 & 157.5 & 0.0 & 49.0 & 42.25 \\
\hline 44 & 0.0 & 0.0 & 0.0 & 0.0 & 0.0 & 0.0 & 0.00 \\
\hline 45 & 0.0 & 0.0 & 0.0 & 0.0 & 0.0 & 0.0 & 0.00 \\
\hline
\end{tabular}




\begin{tabular}{|c|c|c|c|c|c|c|c|}
\hline 46 & 0.0 & 0.0 & 42.0 & 0.0 & 0.0 & 42.0 & 14.00 \\
\hline 47 & 0.0 & 0.0 & 42.0 & 0.0 & 0.0 & 42.0 & 14.00 \\
\hline 48 & 0.0 & 0.0 & 0.0 & 0.0 & 0.0 & 0.0 & 0.00 \\
\hline 49 & 0.0 & 0.0 & 0.0 & 0.0 & 0.0 & 0.0 & 0.00 \\
\hline 50 & 42.0 & 157.5 & 0.0 & 0.0 & 80.5 & 0.0 & 46.67 \\
\hline 51 & 455.0 & 252.0 & 80.5 & 49.0 & 700.0 & 49.0 & 264.25 \\
\hline 52 & 0.0 & 0.0 & 0.0 & 0.0 & 0.0 & 0.0 & 0.00 \\
\hline 53 & 385.0 & 840.0 & 252.0 & 455.0 & 385.0 & 252.0 & 428.17 \\
\hline 54 & 0.0 & 0.0 & 0.0 & 0.0 & 0.0 & 0.0 & 0.00 \\
\hline 55 & 0.0 & 0.0 & 0.0 & 0.0 & 0.0 & 0.0 & 0.00 \\
\hline 56 & 0.0 & 0.0 & 0.0 & 0.0 & 0.0 & 0.0 & 0.00 \\
\hline 57 & 0.0 & 0.0 & 42.0 & 0.0 & 0.0 & 42.0 & 14.00 \\
\hline 58 & 49.0 & 0.0 & 0.0 & 0.0 & 0.0 & 0.0 & 8.17 \\
\hline 59 & 0.0 & 0.0 & 0.0 & 0.0 & 0.0 & 0.0 & 0.00 \\
\hline 60 & 157.5 & 80.5 & 42.0 & 49.0 & 157.5 & 49.0 & 89.25 \\
\hline 61 & 0.0 & 5.0 & 5.0 & 0.0 & 0.0 & 0.0 & 1.67 \\
\hline 62 & 157.5 & 252.0 & 49.0 & 157.5 & 252.0 & 157.5 & 170.92 \\
\hline 63 & 0.0 & 0.0 & 0.0 & 80.5 & 0.0 & 0.0 & 13.42 \\
\hline 64 & 0.0 & 0.0 & 0.0 & 0.0 & 0.0 & 0.0 & 0.00 \\
\hline 65 & 840.0 & 455.0 & 80.5 & 157.5 & 385.0 & 252.0 & 361.67 \\
\hline 66 & 0.0 & 0.0 & 0.0 & 80.5 & 0.0 & 0.0 & 13.42 \\
\hline 67 & 0.0 & 0.0 & 0.0 & 0.0 & 0.0 & 0.0 & 0.00 \\
\hline 68 & 5.0 & 157.5 & 49.0 & 157.5 & 840.0 & 80.5 & 214.92 \\
\hline 69 & 252.0 & 700.0 & 157.5 & 80.5 & 157.5 & 252.0 & 266.58 \\
\hline 70 & 0.0 & 80.5 & 0.0 & 0.0 & 252.0 & 0.0 & 55.42 \\
\hline 71 & 0.0 & 49.0 & 0.0 & 0.0 & 80.5 & 0.0 & 21.58 \\
\hline 72 & 80.5 & 840.0 & 157.5 & 80.5 & 455.0 & 157.5 & 295.17 \\
\hline 73 & 49.0 & 385.0 & 49.0 & $42 \mathrm{M}$ & 455.0 & 252.0 & 198.33 \\
\hline 74 & 0.0 & 0.0 & 0.0 & 0.0 & 0.0 & 0.0 & 0.00 \\
\hline 75 & 0.0 & 0.0 & 0.0 & 0.0 & 0.0 & 0.0 & 0.00 \\
\hline 76 & 80.5 & 0.0 & 42.0 & 0.0 & 0.0 & 42.0 & 27.42 \\
\hline 77 & 0.0 & 0.0 & 0.0 & 0.0 & 0.0 & 0.0 & 0.00 \\
\hline 78 & 0.0 & 0.0 & 0.0 & 0.0 & 0.0 & 0.0 & 0.00 \\
\hline 79 & 49.0 & 252.0 & 80.5 & 80.5 & 700.0 & 157.5 & 219.92 \\
\hline 80 & 700.0 & 252.0 & 49.0 & 80.5 & 252.0 & 157.5 & 248.50 \\
\hline 81 & 0.0 & 0.0 & 0.0 & 0.0 & 0.0 & 0.0 & 0.00 \\
\hline 82 & 5.0 & 0.0 & 0.0 & 0.0 & 0.0 & 0.0 & 0.83 \\
\hline 83 & 0.0 & 0.0 & 42.0 & 0.0 & 0.0 & 42.0 & 14.00 \\
\hline 84 & 0.0 & 0.0 & 0.0 & 0.0 & 0.0 & 0.0 & 0.00 \\
\hline 85 & 80.5 & 385.0 & 49.0 & 49.0 & 80.5 & 80.5 & 120.75 \\
\hline 86 & 0.0 & 0.0 & 0.0 & 0.0 & 0.0 & 0.0 & 0.00 \\
\hline 87 & 157.5 & 0.0 & 0.0 & 80.5 & 0.0 & 0.0 & 39.67 \\
\hline 88 & 0.0 & 0.0 & 42.0 & 0.0 & 0.0 & 49.0 & 15.17 \\
\hline 89 & 0.0 & 0.0 & 0.0 & 5.0 & 0.0 & 0.0 & 0.83 \\
\hline 90 & 0.0 & 0.0 & 0.0 & 0.0 & 0.0 & 0.0 & 0.00 \\
\hline 91 & 385.0 & 840.0 & 252.0 & 80.5 & 455.0 & 252.0 & 377.42 \\
\hline 92 & 49.0 & 252.0 & 157.5 & 252.0 & 80.5 & 385.0 & 196.00 \\
\hline 93 & 157.5 & 700.0 & 455.0 & 455.0 & 980.0 & 385.0 & 522.08 \\
\hline Morocco & 840.0 & 1120.0 & 700.0 & 1120.0 & 1225.0 & 840.0 & 974.17 \\
\hline LSD at $5 \%$ & 50.741 & 49.896 & 51.076 & 48.971 & 46.007 & 49.994 & \\
\hline
\end{tabular}


Also, during the three growing seasons of the study at the two locations, the tested wheat genotypes were divided into two groups depending on the values of AUDPC. The first group is genotypes with partial resistance which showed the lowest values of AUDPC (less than 300). This group included 47 wheat genotypes which showed AUDPC values ranged from 0 to 294. On the other hand, the second group included seven wheat genotypes i.e. 26 (300.42), 38 (357.00), 53 (428.17), 65 (361.67), 91 (377.42), 93 (522.08), and Morocco (974.17) (Table 6).

\section{DISCUSSION}

The rust pathogens with a high reproductive rate and the ability to spread quickly and evolve new pathotypes rapidly are a major threat to food security (Duveiller et al., 2007). Disease resistant wheat cultivars are considered the main factor in agriculture wheat breeding programs to protect wheat plants from disease infection and consequently from yield loss. To sustain the economic viability of wheat production globally, it is necessary to protect crops from the potentially destructive impact of rusts, including leaf rust. This is the most effective method by identifying and deploying new sources of resistance that can durably mitigate the threat of a dynamic and rapidly evolving pathogen population. Moreover, the discovery of novel sources of resistance with novel genes is a constant challenge and is critical in plant breeding to combat threats to crop production caused by pests. In wheat, gene pyramiding to develop durable leaf rust-resistant cultivars is of paramount importance. Moreover, screening germplasm for resistance sources, hybridization of selected parents, selection as well as evaluation of hybrids, testing, and release of new varieties. This may require artificial epidemics created by the inoculation of pathogens onto the plant population (Alemu, 2019).

The probability of identifying resistant parents and resistant progenies is increased by the availability of a reliable screening methodology and an environment favorable for disease development. Depending on the disease and choice of the type of resistance, the methodology may require simple tests in adult plants (field tests) or even the use of resistance-linked protein and DNA markers. Inclusion of check cultivars for resistance and susceptibility is important to assess the disease pressure and degree of resistance. The choice of field sites with reliable environmental conditions is crucial for progress when selection is to be carried out in field conditions.

Among the 716 wheat genotypes collected globally from CIMMYT, 93 wheat genotypes were selected and evaluated against leaf rust at the adult plant stage under field conditions at Menoufia and Behira locations for three successive growing seasons i.e. 2017/2018, $2018 / 2019$, and $2019 / 2020$ growing seasons. Of the 94 wheat genotypes used in our study, 47 genotypes were found to be resistant to at both of the locations during the three successive growing seasons of the study.

Data on rust incidence were recorded as percentage final rust severity, infection type, the average coefficient of infection (ACI), and relative resistance index (RRI). According to the scale of 0-9 of Aslam (1982) to select resistant wheat genotypes for rust diseases, where RRI = 0 means the genotype is highly susceptible and RRI $=9$ means the genotype is highly resistant. Moreover, for leaf rust, RRI $=5$ or 6 means the genotype is acceptable in its resistance, while RRI = 7 and above means the genotype is desirable in its resistance. For stripe and stem rust, RRI = 6 means the genotype is acceptable in its resistance, while RRI $=7$ and above means the genotype is desirable in its resistance. The RRI assessment in this study is used for the second time in Egypt after El-Orabey et al. (2014) who used this scale for the first time in Egypt to evaluate some promising lines from CIMMYT to select the resistant genotype for rust diseases and this point is the new issue in this study.

Data of this study revealed that only 43 wheat lines showed acceptable RRI for leaf rust during the three successive growing seasons compared with Morocco (check). These wheat lines were found to be resistant to leaf rust disease and can be used in breeding programs to release commercial cultivars as safely production under Egyptian conditions. These results are in agreement with Akhtar et al. (2002); Rattu et al. (2009); Hussain et al. (2010a); Hussain et al. (2010b) and Hussain et al. (2013). Moreover, the results are in line with the work done by Mahmood et al. (2013) who reported that the rust score of Chakwal-50 varied from 5 MR/MS to 30 MS for leaf rust. Also, cv. Chakwal-50 gave RRI value of 7 to 8.6 for leaf rust. The cv. Chakwal-50 has the potential to be approved and released as a new variety. Our results conform with those of El-Orabey et al. (2014) who found that out of sixteen CIMMYT promising lines, seven lines, i.e. 1, 2, 7, 8, 10, 11, and 15 were found to be resistant to rust diseases and showed 
acceptable/desirable relative resistance index (RRI) during the two seasons 2012/13 and 2013/14.

The 43 tested wheat promising lines which were resistant at the two locations during the three growing seasons of the study should be tested for grain yield and other agronomic characters i.e. Days to heading and maturity, plant height $(\mathrm{cm})$, biological yield $(\mathrm{kg})$, straw yield and also flour extraction (\%) and rheological properties to be registered as a new commercial cultivar, also, it must identify the rust resistance genes present in these lines by the molecular marker to know the leaf rust resistance genes and the number of genes present in these lines.

\section{CONCLUSION}

The variability and constant evolution of wheat leaf rust populations exhort huge pressure on wheat breeders and researchers, in general, to be constantly vigilant against the emergence of new rust races. This requires timeous monitoring and collaborative surveillance of changes in the virulence patterns among rust pathogens in each country and across regions. Results of this study were promising and some immune, resistant, and moderately resistant genotypes to Puccinia triticina were identified and they may be used as a resistance genetic source for management of the disease in national programs. Wheat leaf rust in Egypt has caused significant crop loss and resulted in unprecedented costs in chemical control expenditure in epidemic seasons. It can be anticipated that control measures will be largely based on the development and release of resistant cultivars. Breeding for resistance will continue to be based on current awareness of variability in Puccinia triticina, the search for and commercial development of new and effective resistance combinations, and the resolve of the industry to adopt best management practices that minimize disease risk.

\section{REFERENCES}

Akhtar, M. A., I. Ahmad, J. I. Mirza, A. R. Rattu, E. UlHaque, A. A. Hakro and A. H. Jaffery. 2002. Evaluation of candidate lines against stripe and leaf rusts under national uniform wheat and barley yield trial 2000-2001. Asian Journal of Plant Sciences, 1: 450-53.

Alemu, G. 2019. Wheat breeding for disease resistance: Review. Journal of Microbiology and Biotechnology, 4: 1-10.
Anwar, M. J., M. A. Javed, M. W. Jamil, I. Habib, S. Nazir, S. Ur Rehman, M. Z. Iqbal, M. Kamran and M. Ehetisham ul Haq. 2019. Response of wheat genotypes against leaf rust (Puccinia triticina) under field conditions. Plant Protection, 3: 35-39.

Aslam, M. 1982. Uniform Procedure for Development and Release of Improved Wheat Varieties. Mimeograph, ARC: Islamabad, Pakistan.

Bux, H., M. Ashraf, F. Hussain, A. R. Rattu and M. Fayyaz. 2012. Characterization of wheat germplasm for stripe rust (Puccini striiformis f. sp. tritici) resistance. Australian Journal of Crop Science, 6: 116-20.

Daetwyler, H. D., U. K. Bansal, H. S. Bariana, M. J. Hayden and B. J. Hayes. 2014. Genomic prediction for rust resistance in diverse wheat landraces. Theoretical and Applied Genetics, 127: 1795-803.

Duveiller, E., R. P. Singh and J. M. Nicol. 2007. The challenges of maintaining wheat productivity: Pests, diseases, and potential epidemics. Euphytica, 157: 417-30.

El-Orabey, W., R. Omara and M. Abou-Zeid. 2018. Diversity and virulence dynamics within Puccinia triticina populations in Egypt. Journal of Plant Protection and Pathology, 9: 735-45.

El-Orabey, W., K. Ragab and M. El-Nahas. 2014. Evaluation of some bread wheat promising lines against rust diseases. Egyptian Journal of Phytopathology, 42: 83-100.

El-Orabey, W. M., N. I. Abd El-Malik, M. A. Ashmawy and M. A. Abou-Zeid. 2017. Reduction in grain yield caused by leaf rust infection in seven Egyptian wheat cultivars. Minufiya Journal of Plant Protection, 2: 71-81.

Huerta-Espino, J., R. P. Singh, S. Germán, B. D. McCallum, R. F. Park, W. Q. Chen, S. C. Bhardwaj and H. Goyeau. 2011. Global status of wheat leaf rust caused by Puccinia triticina. Euphytica, 179: 14360.

Hussain, M., L. H. Akhtar, M. Rafiq, M. Z. Aslam, A. H. Tariq, M. Aslam, M. Arshad and S. Ahmad. 2010a. Mairaj-08: New wheat (Triticum aestivum) variety released for general cultivation under normal and late planting in Punjab province (Pakistan). International Journal of Agriculture and Biology, 12: 341-47.

Hussain, M., L. H. Akhtar, A. H. Tariq, M. Rafiq and M. Nasim. 2010b. Manthar-03: A high-yielding 
cultivar of wheat released for general cultivation in southern Punjab. Pakistan Journal of Agricultural Sciences, 47: 375-82.

Hussain, M., M. Rafiq, L. H. Akhtar, A. H. Tariq, S. Ahmad, M. Z. Aslam, M. A. Nadeem and M. Zubair. 2013. Release of high yielding wheat variety AaS-2011 resistance to stem rust (Ug-99) in Pakistan. Journal of Animal and Plant Sciences, 23: 1115-24.

Jin, Y. and R. P. Singh. 2006. Resistance in U.S. wheat to recent eastern African isolates of Puccinia graminis f. sp. tritici with virulence to resistance gene Sr31. Plant disease, 90: 476-80.

Mahmood, A., M. A. Mian, M. Ihsan, M. Ijaz, G. Rabbani and M. S. Iqbal. 2013. Chakwal-50: A high yielding and disease resistant wheat variety for rainfed region. Journal of Animal and Plant Sciences, 23: 833-39.

McIntosh, R. A., C. R. Wellings and R. F. Park. 1995. Wheat Rusts: An Atlas of Resistance GenesCSIRO Publishing. Australia. pp. 213.

Pandey, H. N., T. C. M. Menon and M. V. Rao. 1989. A simple formula for calculating area under disease progress curve. Rachis, 8: 38-39.

Peterson, R. F., A. B. Campbell and A. E. Hannah. 1948. A diagrammatic scale for estimating rust intensity on leaves and stems of cereals. Canadian Journal of Research, 26: 496-500.

Rattu, A. R., M. A. Akhtar, M. Fayyaz and M. Bashir. 2009. Screening of wheat against yellow and leaf rusts under NUWYT and NWDSN and wheat rust situation in Pakistan during 2006-2007Crop Diseases Research Programmes, NARC. Islamabad, Pakistan.
Sapkota, S., Y. Hao, J. Johnson, B. Lopez, D. Bland, Z. Chen, S. Sutton, J. Buck, J. Youmans and M. Mergoum. 2018. Genetic mapping of a major gene for leaf rust resistance in soft red winter wheat cultivar AGS 2000. Molecular Breeding, 39: 1-11.

Shahin, S. I. and W. M. El-Orabey. 2016. Assessment of grain yield losses caused by Puccinia triticina in some Egyptian wheat genotypes. Minufiya Journal of Agricultural Research, 41: 29-37.

Singh, R. P., J. Huerta-Espino and A. P. Roelfs. 2002. Wheat for Bread and other Foods. In: B. C. Curtis, S. Rjaram and H. G. Macpherson (eds.), Bread Wheat: Improvement and Production. FAO: Rome, Italy.

Snedecor, G. W. and W. G. Cochran. 1967. Statistical Methods. Iowa State University Press: Ames, Iowa, U.S.A.

Stakman, E. C., D. Stewart and W. Loegering. 1962. Identification of physiologic races of Puccinia graminis var. triticiUnited States Department of Agriculture. Washington, USA.

Stubbs, R. W., J. M. Prescott, E. E. Saari and H. J. Dubin. 1986. Cereal Disease Methodology Manual. Centro Internacional de Mejoramiento de Maiz y Trigo (CIMMYT): México.

Tervet, I. W. and R. C. Cassell. 1951. The use of cyclone separators in race identification of cereal rusts. Phytopathology, 41: 286-90.

Tyagi, S., R. R. Mir, H. Kaur, P. Chhuneja, B. Ramesh, H. S. Balyan and P. K. Gupta. 2014. Marker-assisted pyramiding of eight QTLs/genes for seven different traits in common wheat (Triticum aestivum L.). Molecular Breeding, 34: 167-75.

\section{CONFLICT OF INTEREST}

The authors declare that they have no conflicts of interest.

\section{AUTHORS CONTRIBUTIONS}

Walid El-Orabey wrote the first draft of the article and finalized it with contributions by Hosam Awad, Sabry Shahin, and Yasser El-Gohary.

Publisher's note: EScience Press remains neutral with regard to jurisdictional claims in published maps and institutional affiliations.

Open Access This article is licensed under a Creative Commons Attribution 4.0 International License, which permits use, sharing, adaptation, distribution and reproduction in any medium or format, as long as you give appropriate credit to the original author(s) and the source, provide a link to the Creative Commons license and indicate if changes were made. The images or other third-party material in this article are included in the article's Creative Commons license, unless indicated otherwise in a credit line to the material. If material is not included in the article's Creative Commons license and your intended use is not permitted by statutory regulation or exceeds the permitted use, you will need to obtain permission directly from the copyright holder. To view a copy of this license, visit http://creativecommons.org/licenses/by/4.0/. 